\title{
Deformability of a woven fabric modified with in-situ grown nanofibres
}

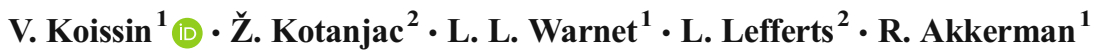

Received: 16 August 2016 / Accepted: 5 November 2017 / Published online: 20 November 2017

(C) The Author(s) 2017. This article is an open access publication

\begin{abstract}
The deformability of a textile fabric (as received, or with in-situ grown carbon nano-fibres, or with a thermal treatment) is studied experimentally using bending, compression, in-plane shear, and friction. These tests reveal that the in-situ grown carbon nano-fibres can significantly deteriorate the fabric deformability, except for a low mass fraction. Therefore, the optimal mass fraction should be chosen for a particular production case, to obtain a compromise between improved strength of a composite part (expected to increase after the nano-fibre growth) and decreased deformability of the preform.
\end{abstract}

Keywords Fabrics $\cdot$ Carbon fibres $\cdot$ Chemical vapor deposition $\cdot$ Nano-fibres $\cdot$ Deformability

\section{Introduction}

When producing or modelling three-dimensionally shaped textile composites, deformability of the fabric is a crucial is-

L. L. Warnet

L.Warnet@utwente.nl

1 Production Technology Group, Department of Mechanical Engineering, University of Twente, 7500AE, Enschede, The Netherlands

2 Catalytic Processes and Materials Group, Department of Chemical Engineering, University of Twente, 7500AE, Enschede, The Netherlands sue. Even for a flat part, one of the deformability properties compliance to the through-thickness compression - is very important to obtain the target fibre content. For a curved part, possible issues involve also bending, shearing, friction, tension, prediction of the fibre orientation, wrinkling, etc. This has received attention of many researchers, e.g. [1]. However, due to various types of fabrics, yarns, fibres, as well as their numerous combinations and treatments, this research line seems to be endless.

One of these possible treatments applied to carbon-fibre fabrics is the in-situ growth of carbon nanotubes (CNTs) or nanofibres (CNFs) on the surface of microfibres [2]. This "grafting" creates a "forest" on the fibre surface, generally acting as the well-known whiskerizing but on a lower scale. On one hand, this modification is expected to improve the fibre-matrix interface and crack bridging and, consequently, the damage resistance [3-5]. On the other hand, the deformability is foreseen to be altered by numerous interfibre links as well as by the extra amount of carbon introduced into the fabric $[6,7]$. If the deformability is indeed altered significantly, then in some production techniques (e.g. in the low-pressure vacuum infusion) this disadvantage can overshadow all possible improvements in strength.

According to the authors' knowledge, deformability of nano-grafted fabrics has almost not been addressed in the past, except for the study reported in [8-10] focused on fabric compressibility at a relatively low pressure. The present study aims at a broader experimental program. Some of the used test methods are more useful for thermoplastics (e.g. compressibility at a high pressure), while others (bending, shear, friction tests) are also important for a broader set of production techniques. The study also aims to answer two main questions: a) what is the typical magnitude of "stiffening" introduced by insitu grown CNFs into a carbon-fibre fabric and b) what are possible reasons for this behavior? 


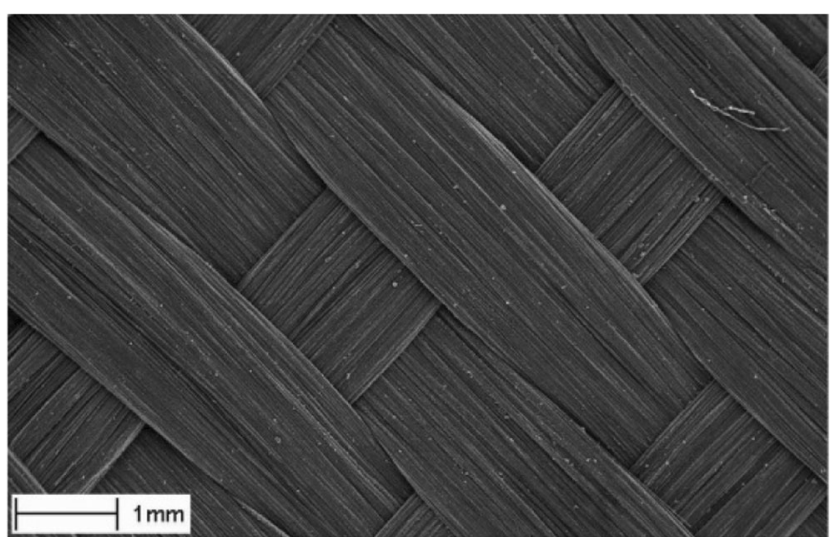

Fig. 1 Unit-cell structure of the used fabric. The weft yarns float over 4 warp yarns

\section{Materials}

A typical woven carbon reinforcement (CD 0286 supplied by Ten Cate AC) is used as the base material. This 5-harness satin weave, Fig. 1, is made of $3 \mathrm{~K}$ non-twisted tows of Torayca T300 J $7 \mu \mathrm{m}$ diameter fibres. The areal weight is $285 \mathrm{~g} / \mathrm{m}^{2}$. Nano-grafted fabrics $(26 \times 31 \mathrm{~cm}$ sheets $)$ were manufactured using the Chemical Vapor Deposition (CVD) technique [11].

For comparison, the tests presented below were performed not only for the base and grafted fabrics but also for a number of reference configurations. The total list is the following:

1) "Sized" fabric (TenCate CD 0286050000 0000) having the original Torayca standard sizing by about $1 \%$ of the fabric, by weight [12]. Its chemical composition is not clear; it is based on an epoxy that should solidify when impregnating the preform with another epoxy resin. A typical surface is shown in Fig. 2, which reveals that many fibres are virtually bonded together by the sizing.

2) "Base" fabric (TenCate CD 0286050000 8212) which was partially de-sized for better compatibility with the PPS matrix. There are two methods: a) heating at 330
$430{ }^{\circ} \mathrm{C}$ for $2-4 \mathrm{~h}$ or b) heating at $380-520{ }^{\circ} \mathrm{C}$ for $15 \pm 5 \mathrm{~min}$ and then at $190-250{ }^{\circ} \mathrm{C}$ for $3-5 \mathrm{~h}$. It is not known which method was employed for the fabric used in the present study but it is important that "...the carbon fibres remain unaffected and the epoxy material is aged or neutralized and loses its tacky character" [13]. This means that the sizing is just "neutralized" but not removed completely as seen in Fig. 3a and also further in Fig. 4. This fabric is used as the base material for the cases listed below, including the case of CNF growth.

3) "Desized" fabric prepared by heating the "base" fabric up to $600{ }^{\circ} \mathrm{C}$ in $\mathrm{N}_{2}$ atmosphere, for about $30 \mathrm{~min}$, without the Ni catalyst and CNF growth. This mimics the thermal treatment during $\mathrm{Ni}$ deposition and/or CNF growth. Weighing of the samples before and after this treatment shows a $0.5 \%$ weight loss. As seen in Fig. 4 , the sizing is again not removed completely but rather carbonized, as supported by chemical analysis [14]. The fabric becomes more compliant by touch; its yarns become very "loose" and easily disintegrate into single fibres after cutting.

4) "Ni-loaded" fabric prepared by spraying the "base" fabric with an acetone solution containing $0.25 \mathrm{wt} . \%$ nickel-nitrate. Then it is dried at room temperature for $10 \mathrm{~min}$ and placed in a reactor [11], where $\mathrm{Ni}\left(\mathrm{NO}_{3}\right)_{2}$ is reduced with hydrogen (at $600{ }^{\circ} \mathrm{C}$, during $60 \mathrm{~min}, \mathrm{~N}_{2}: \mathrm{H}_{2}=70: 30$ ) to obtain Ni particles, Fig. 5, needed for CNF growth. This increases the fabric weight by about $0.7 \%$ but the fabric is now even yet more compliant by touch than the "desized" one, maybe due to a chemical reaction of sizing with the Ni salt or dissolution in acetone.

It is worth to note that this Ni-loaded fabric is the best reference to be compared with a nano-grafted fabric (in the sense of mechanical properties, chemical composition, etc.). This shows the full effect of the grown CNFs, while comparison with the material just taken from a roll involves the effect of sizing.
Fig. 2 Original Torayca sizing on fibres (a) and their larger magnification (b)
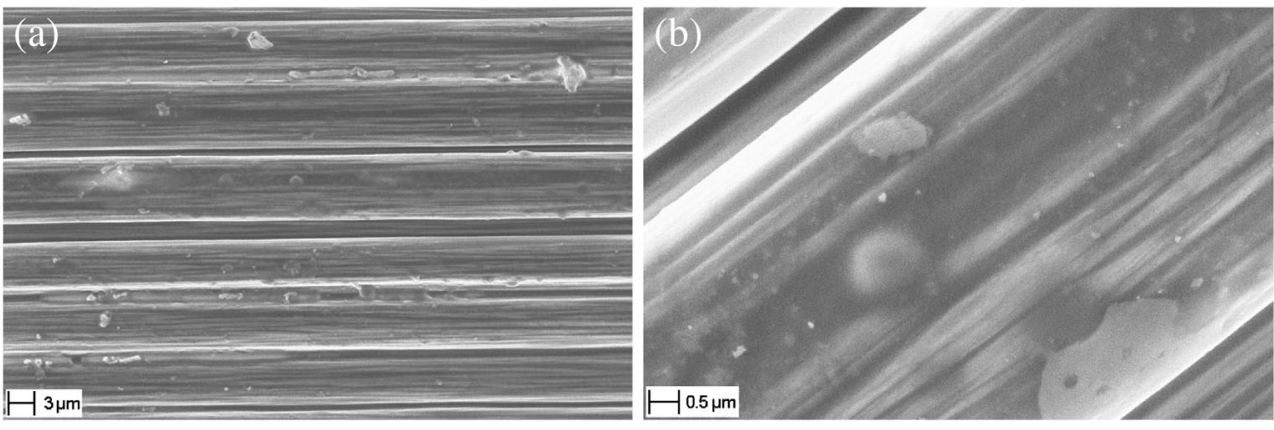
Fig. 3 Fibres in the "base" fabric, small (a) and large (b) magnifications
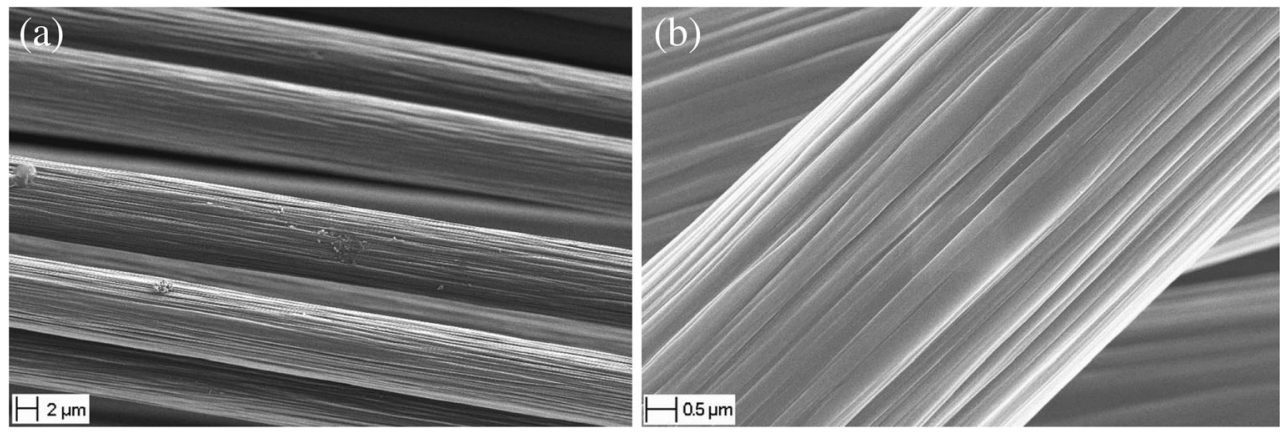

5) "Nano-grafted" fabrics are the same as the "Ni-loaded" ones but subsequently CNFs are grown in a hydrocarbon gas mixture (at $600{ }^{\circ} \mathrm{C}$, during 30-45 min, $\mathrm{N}_{2}: \mathrm{C}_{2} \mathrm{H}_{4}: \mathrm{H}_{2}=75: 20: 5$ ), as described in [11]. The CNF weight fraction (wt.\%) is controlled by varying the reaction time. The wt.\% is calculated as $\left(\mathrm{M}_{\mathrm{N}}-\mathrm{M}_{0}\right) / \mathrm{M}_{\mathrm{N}}$, where $\mathrm{M}_{0}$ and $\mathrm{M}_{\mathrm{N}}$ are the initial and after-grafting weights of the specimen, respectively. This value accounts not only for CNFs but also for $0.7 \mathrm{wt} . \%$ of Ni mentioned above and $0.5 \mathrm{wt} . \%$ of "desizing".

One to three fabrics were prepared for every test case in the list above.

The apparent compliancy of the fabric depends on the CNF-loading. The fabric is more flexible by touch than the "base" fabric in case of CNF-loading of typically $1 \mathrm{wt} \%$. In that case, the yarns can be easily deformed and disintegrated. Higher CNF-loadings induce higher apparent bending stiffness, as well as better integrity of yarns and the textile structure, since the growth is both intra-yarn and inter-yarn. For example, a $15 \mathrm{wt} . \%$ case shows a prominent entanglement of adjacent yarns, and a certain force is needed to split them by pincers. Even higher CNF-loading, e.g. 20-40 wt.\%, changes the fabric into a sort of "carbon-carbon composite" which by touch is like a rigid cardboard. Of course, such large wt.\% are not practical in composite production and are studied below merely as ultimate cases.

Figure 6 shows typical SEM images, where the overall distribution of grafting on the fibres is mostly uniform. However, there is no local uniformity: CNFs are randomly shaped and strongly entangled. There are large CNFs with diameters about $100 \mathrm{~nm}$, thin CNFs with diameters 20 $50 \mathrm{~nm}$, and even thinner 5-20 nm diameter CNFs [8]. The second type is seen well in Fig. 6b, while the other two are well-presented in Fig. 7b.

It was not investigated in depth in this study but it seems that larger CNFs appear only after a relatively high growth (say, more than 3-4 wt.\%). Several SEM pictures taken for a lower growth $(0.7 \mathrm{wt} . \%)$ show only thin $5 . .20 \mathrm{~nm}$ CNFs.

\section{Bending}

All tests reported in this paper are conducted at the ambient conditions $\left(\sim 22^{\circ} \mathrm{C}, \sim 70 \% \mathrm{RH}\right)$.

The Pierce cantilever test is the simplest procedure to estimate the fabric drapability. In the present study, a narrow (2.5$3.5 \mathrm{~cm}$ wide and $30 \mathrm{~cm}$ long) strip of fabric is rigidly clamped and subjected to the action of its own weight. One specimen was tested for every configuration. The warp yarns (that is zero direction, Fig. 1) are oriented along the strip at its upper (convex) side.

The curved geometry is captured by a camera equipped with a low-distortion lens. The picture is committed into a Matlab applet collecting coordinates of a number of points chosen manually along the curve. Typical bent shapes for different span lengths are shown in Fig. 8, visually revealing a prominent effect of grafting on the bending stiffness.

It should be noted that, in spite of its apparent simplicity, a correct execution of this test for textiles is not an easy task. For example, it is difficult to achieve a zero slope in
Fig. 4 "Desized" fibres, small (a) and large (b) magnifications
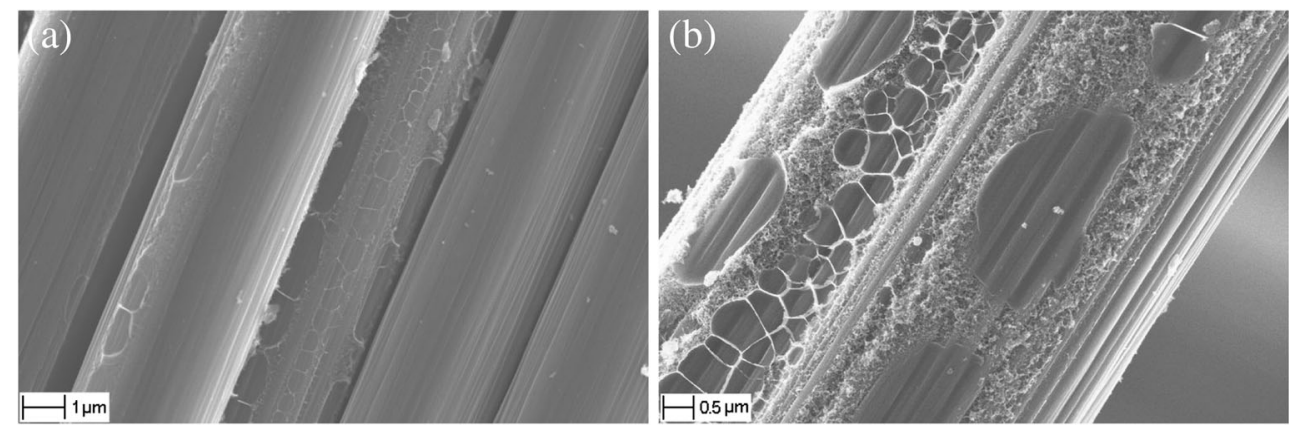
Fig. 5 Ni-loaded fibres, small (a) and large (b) magnifications
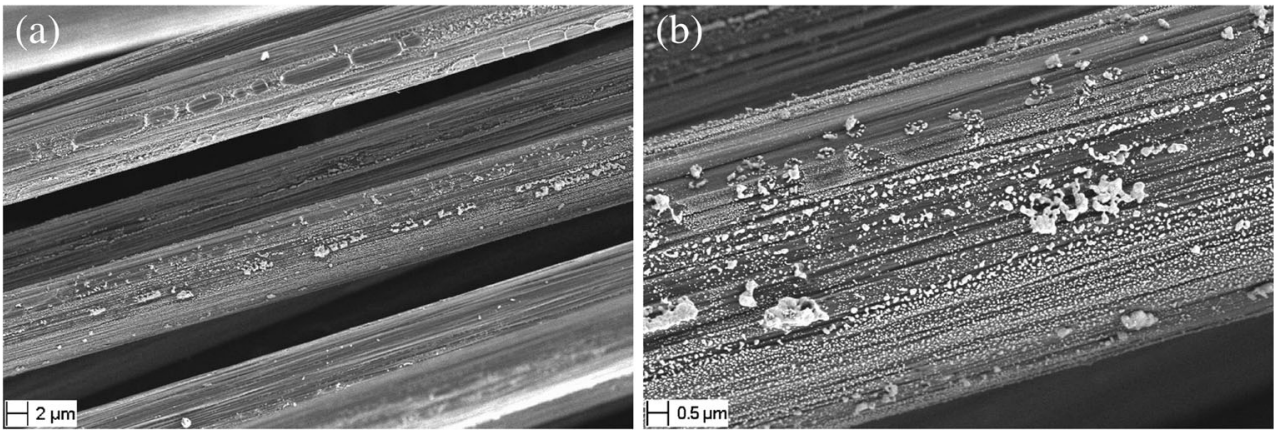

the clamp. In addition, compaction of the fabric is likely to occur in the clamp, affecting the local thickness. Also, even small internal forces cause a torsional deformation of the strips (it was kept minimal in the present tests). In the present tests, these internal stresses do not only rise due to handling and cutting but also due to grown CNFs which "inflate" the yarns.

For the "desized" fabric, or Ni-loaded one, or that containing a small amount of CNFs, the specimen preparation is also not easy. This is because, as noticed above, the fibres become very "loose" and hardly allow cutting and handling good specimen geometry.

Nevertheless, this test can still give a qualitative estimation of the bending stiffness, as demonstrated in Fig. 8. An approximate quantitative analysis is also possible. Here, we employ a numerical solution, [15], subjected to the condition of minimal least squares of the differences with the test data, having a constant bending stiffness as the design variable.

The calculated results are collected in Table 1 which shows that grown CNFs significantly increase the bending stiffness, while "burning" or Ni salt treatment decreases it. Therefore it may be suggested that a small (say, $0.5-1.5$ wt.\%) amount of CNFs could still give a bending stiffness smaller than that of the sized fabric.

For the non-grafted specimens, which are relatively flexible and prone to concentrate their bending near the clamp, 200 and $240 \mathrm{~mm}$ span lengths usually result in a reduced "average" bending stiffness, while $100 \mathrm{~mm}$ and $150 \mathrm{~mm}$ spans result in larger bending stiffness. This presumably indicates nonlinearity in the areas of high curvature.

\section{Low-pressure compressibility}

Out-of-plane compression test were performed using a rheometer equipped with a $50 \mathrm{~N}$ load cell and a $25 \mathrm{~mm}$-diameter top loading plate. This provides a contact pressure up to 1 bar $(0.1 \mathrm{MPa})$ and thus the tests can mimic the fabric compression during vacuum infusion. The bottom plate is wider and wellaligned to the top plate, to ensure their parallelism. Their possible very small misalignment is compensated by a relatively large specimen thickness and compliance.

The specimens have a $50 \times 50 \mathrm{~mm}$ in-plane size and are composed of 6 plies, $\left[(0 / 90)_{3}\right]_{\mathrm{s}}$ lay-up. To check the effect of lubrication, the tests are done either for dry or wet fabrics. The latter were prepared by wetting the specimens with LV1564SP resin (without hardener). They were kept in a free state for about $3 \mathrm{~h}$, to allow the resin to infiltrate inside the yarns.

The first loading was done without a specimen, to check for the rig compliance. Then three successive compression cycles were applied to every specimen, at a $0.25 \mathrm{~mm} / \mathrm{min}$ displacement rate, to measure its initial (the 1st cycle) and "set" (other two cycles) compressibility. Typical 1 st cycle results under wet conditions are shown in Fig. 9, presenting the pressure vs. the fibre volume fraction $\left(V_{f}\right)$. The latter is calculated in percents as

$V_{f}=100 * W * N /\left(t^{*} \rho\right)$,

where $W$ is areal weight $\left(285 \mathrm{~g} / \mathrm{m}^{2}, \mathrm{CNFs}\right.$ are not accounted for), $N$ is the number of plies, $t$ is the gap between the loading plates (corrected for compliance), and $\rho$ is the fibre density $\left(1.78 \mathrm{~g} / \mathrm{cm}^{3}\right)$.
Fig. 6 A 7.1 wt.\% grafted fibre (a) and typical CNFs (16 wt.\% case, $\mathbf{b}$ )
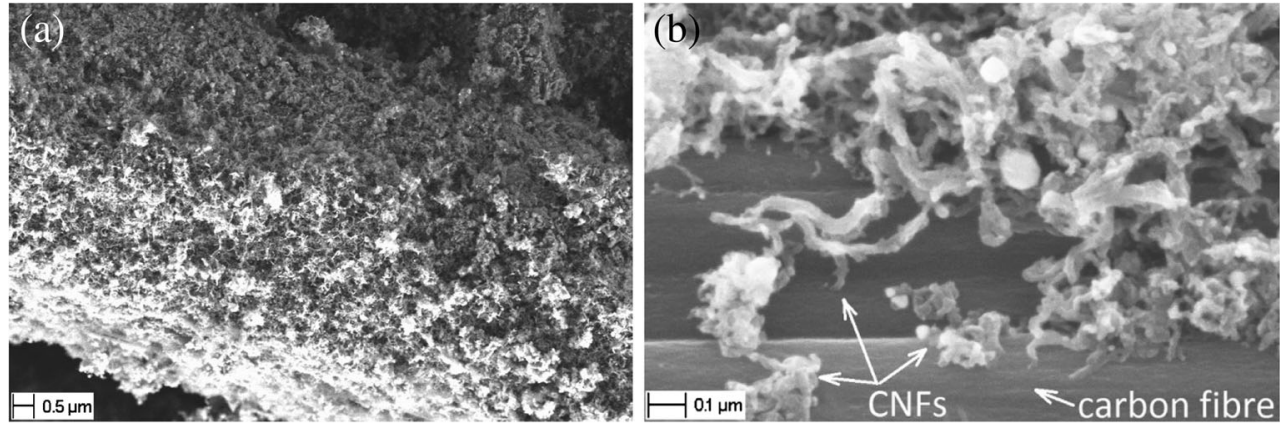
Fig. 7 Typical CNF attachments to the fibres ( $\approx 5 \mathrm{wt} . \%$ case)
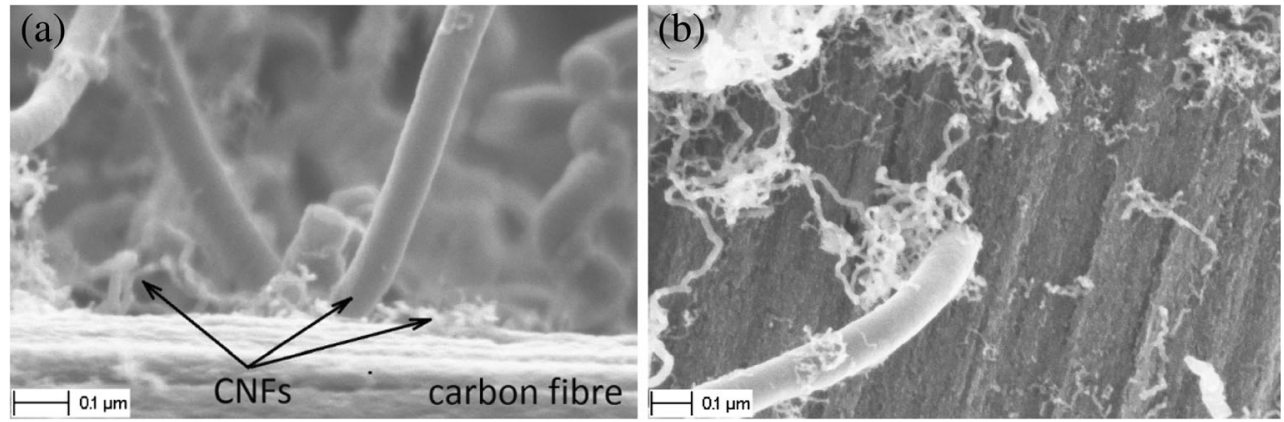

Generally these figures reveal a consistent decrease of compressibility (or, in other words, increase of the fabric thickness) for a given pressure after grafting. The Ni-loaded specimen shows the highest compressibility, even better than the "desized" fabric. This might possibly be explained by additional "removing" of sizing by acetone and $\mathrm{Ni}\left(\mathrm{NO}_{3}\right)_{2}$. The responses of the base and sized fabrics are naturally between these of the "desized" and grafted ones.

The effect of wetting is illustrated in Fig. 10a showing the ratio of Vf in the wet and dry fabrics. As opposed to preliminary expectations, the epoxy does not always act as a lubricant. For the fabrics without or with a small amount of CNFs, it rather gives additional resistance, thus decreasing $\mathrm{Vf}$, at least for the considered pressure range. This can be explained by a "sticky" effect of the epoxy adhering the neighboring fibres; viscosity can also play a role.

As for the fabrics containing large $\mathrm{CNF}$ wt.\%, the effect of wetting is not consistent and not yet understood by the authors: while for the 1.4 and $6.3 \mathrm{wt} . \%$ cases Vf significantly decreases after wetting, the $3.8 \mathrm{wt} \%$ case shows quite inverse effect, and the $8.6 \mathrm{wt} . \%$ case shows no effect at all. The discrepancy is so prominent that the "wet" $1.4 \mathrm{wt}$.\% case shows a lower Vf than the "wet" $0.5 \mathrm{wt} . \%$ case, although they are in an inverse relation in the dry state. The same unexpected relation happens for the 6.3 and $3.8 \mathrm{wt} . \%$ cases.
Returning to Fig. 9, it is worth to note that even the "wet" Vf does not always approach the value measured for vacuuminfused epoxy composites made of the same fabric [16]. This can be due to creep effects not measured in the compression tests. To check this, the fabrics -4 plies, $\left[(0 / 90)_{2}\right]_{\mathrm{s}}$ lay-up - are compressed up to 0.8 bar for half an hour. This pressure level is used because it is not recommended to use the maximal load cell capacity for a long time.

The creep test results shown in Fig. 10b reveal a prominent about $2.5 \%$ for "wet" grafted fabrics - increase of Vf already within a few minutes of the creep monitoring. However, this covers only about a half of the discrepancy, Fig. 9, between Vf measured in the present tests and Vf for composites reported in [16]. It can be suggested that another part of the discrepancy is partially covered by the long-time creep (say, from $30 \mathrm{~min}$ to the vitrification time). In addition, the relative creep magnitude can be slightly larger at 1 bar pressure than at 0.8 bar.

It is interesting to note a relatively larger creep in $\mathrm{Ni}$-loaded fabric, while addition of sizing or CNFs tends to lead to a lower creep. It may be suggested that the sizing or CNF "forests" somehow retain the absorbed resin thus limiting its squeezing out of the specimen. Generally, the squeezing seems to play a significant role, so the magnitude of creep might probably be smaller for larger specimens having smaller perimeter/surface ratio.
Fig. 8 Bent shapes for the base (left) and 6.5 wt.\% grafted (right) fabrics
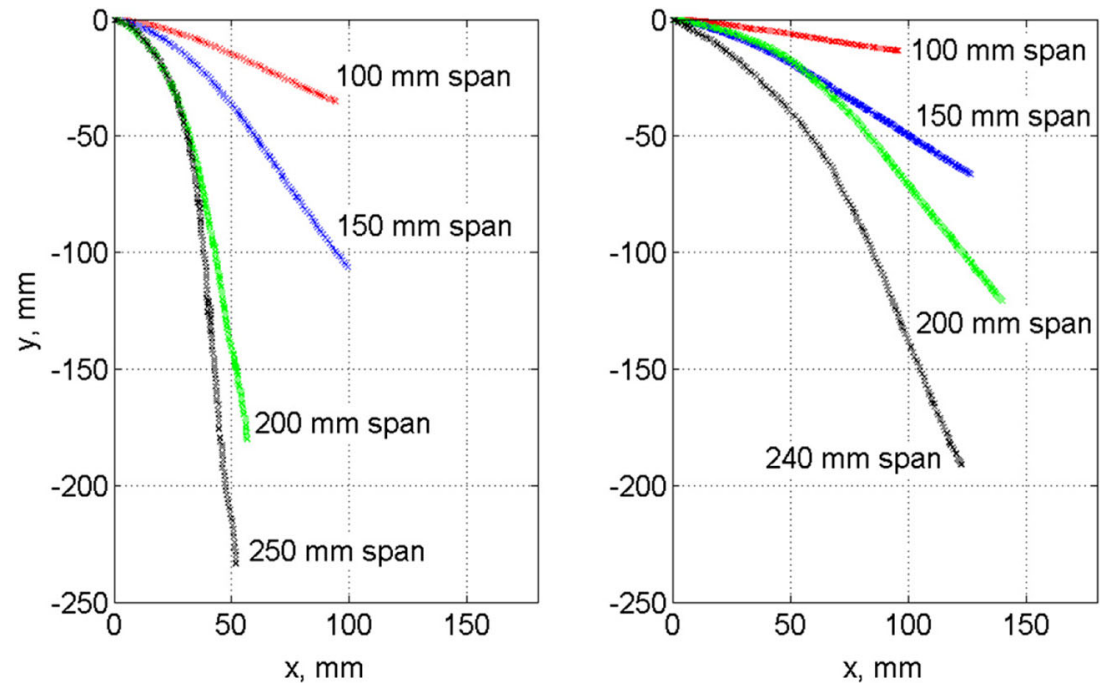
Table 1 Bending stiffness, $\mathrm{N} \cdot \mathrm{m}^{2} \times 10^{-6}$

\begin{tabular}{llrll}
\hline Span length, mm: & 100 & 150 & 200 & 240 \\
\hline Sized & 40.3 & 35.3 & 22.1 & 20.9 \\
Base & 33.4 & 32.2 & 15.6 & 17.2 \\
Desized & 11.9 & 12.2 & 12.5 & 5.9 \\
Ni-loaded & 11.9 & 6.3 & 3.2 & 2.1 \\
Grafted 6.5 wt.\% & 48.1 & 53.7 & - & 55.8 \\
Grafted 11 wt.\% & - & 556.6 & 702.6 & 1031.1 \\
Grafted 39 wt.\% & - & 780.1 & 1240.0 & - \\
\hline
\end{tabular}

Although only the 1st compression is interesting from the production point of view, two subsequent ones are measured also, to estimate the irreversible deformation. Before the repeated loading, the specimens are held in a stress-free state for about $5 \mathrm{~min}$ (dry fabric) or half an hour (wet fabric), to allow the internal stresses to be balanced (spring-back).

Typical results are presented in Fig. 11. The grafted fabrics, except for the $1.4 \mathrm{wt} \%$ case, show the lowest differences between the succeeding loadings; this effect may probably be attributed to a better "setting", due to nano-links crushed and entangled during the first loading. For other fabrics the difference is more pronounced but does not exceed a $2 \%$ deviation from the preceding cycle. The $3 \mathrm{rd}$ compression gives even smaller difference, less than $1 \%$.

\section{High-pressure compressibility}

To study the high-pressure "wet" compressibility, $60 \times 60 \mathrm{~mm}$ preforms - 12 plies, [(0/90) $]_{\text {s }}$ lay-up - are hot-pressed with 13 Fortron 214C $160 \mu \mathrm{m}$ PPS films. This small press is attached to a universal testing machine and consists of two thick compression plates. Each plate is equipped with two cylindrical heating elements and a thermocouple. Four side plates are attached to the bottom press plate, to avoid too large squeezing of matrix.
After the production temperature $\left(325^{\circ} \mathrm{C}\right)$ is held for about $10 \mathrm{~min}$, the specimen is pressed at a $0.25 \mathrm{~mm} / \mathrm{min}$ rate and then cooled down to $150{ }^{\circ} \mathrm{C}$ while keeping the pressure level. This step takes about 20 min since no forced cooling is implemented. Examples of the load-time and temperaturetime regimes are shown in Fig. 12. Parameters of the pressings - two base laminates at different pressures and two laminates with different graftings - are listed in Table 2. The thickness given here was measured after cooling to room temperature.

As seen in Table 2, four plates were produced, two with about $57 \%$ and two with about $62 \% \mathrm{Vf}$, each pair consisting of one with and one without CNFs. Worth to note that $3.8 \mathrm{wt} \%$ grafted fabric, specimen \#4, requires a twice higher pressure, to have almost the same thickness as the base fabric in specimen \#2. On the other hand, the fabric having a low $-0.9 \mathrm{wt} . \%$ - CNF content, plate \#3, does not require a change of pressure. This agrees well with the low-pressure compression of 0.5 or $1.4 \mathrm{wt} . \%$ fabrics presented above.

Results for Vf vs. pressure are presented in Fig. 13a, also in comparison with the compression curve for a dry "base" fabric measured with the same set-up. Here, the thickness (and therefore Vf) was measured in-situ and then corrected for the compliance measured also in-situ at the same testing regime (temperature, displacement rate,...).

It is seen in Fig. 13a that the grafting, as well as the highviscosity PPS melt, significantly increases the compression resistance and, therefore, decreases the transient value of $\mathrm{Vf}$ (shown with lines) which is lower than the resulting "set" Vf measured for the produced composites (shown with dots). The through-thickness shrinkage due to PPS solidification should also play a role in producing this difference.

Therefore, the creep effect should be again accounted for, as shown in Fig. 13b, where the thickness is monitored from the moment of setting a 10 bar pressure. The effect is more pronounced than for the low-viscosity epoxy resin and consists a $20 \%$ of the transient value of $\mathrm{Vf}$. If this correction for the creep is applied, Vf approaches well its value for the solid composite plates, Table 2.
Fig. $9 V_{f}$ vs. pressure in "wet" fabrics with different treatment or CNF wt.\%, for the 1st cycle of compression. Solid dots show data for epoxy-based vacuuminfused composites measured in [16] (a)

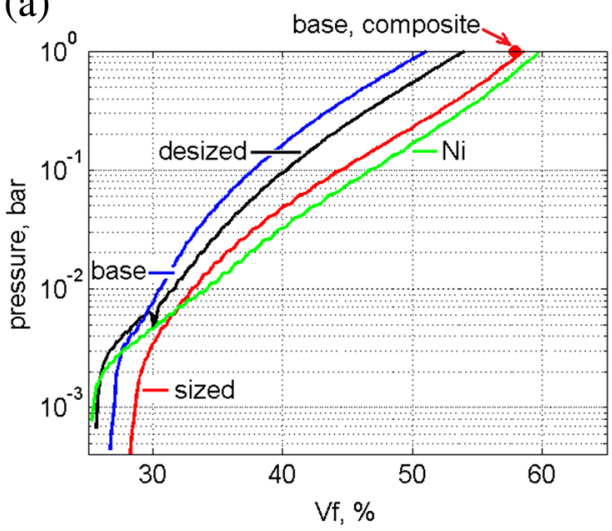

(b)

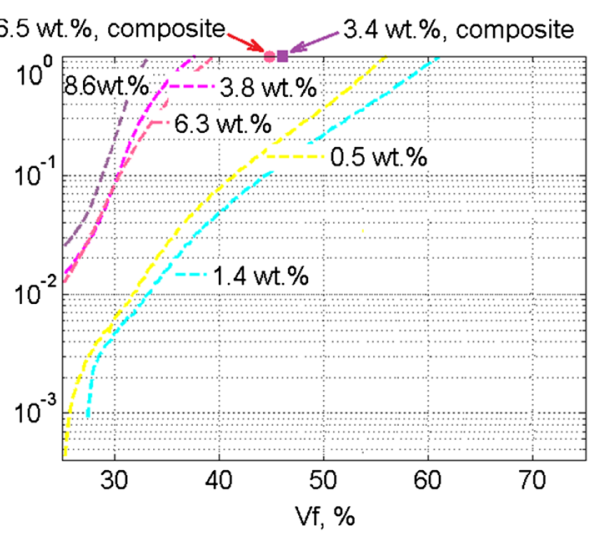


Fig. 10 a Ratio of Vf for wet fabric to that for dry fabric, vs. pressure, for the 1st compression. b Creep behavior of wet fabrics under a 0.8 bar pressure, after the 1 st compression

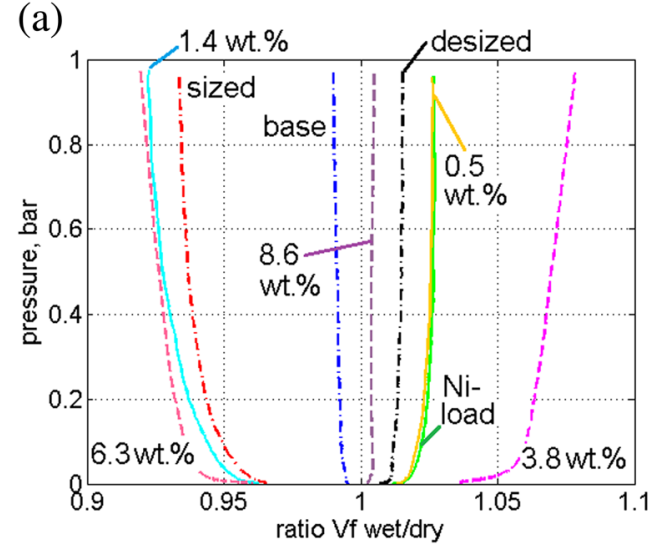

(b)

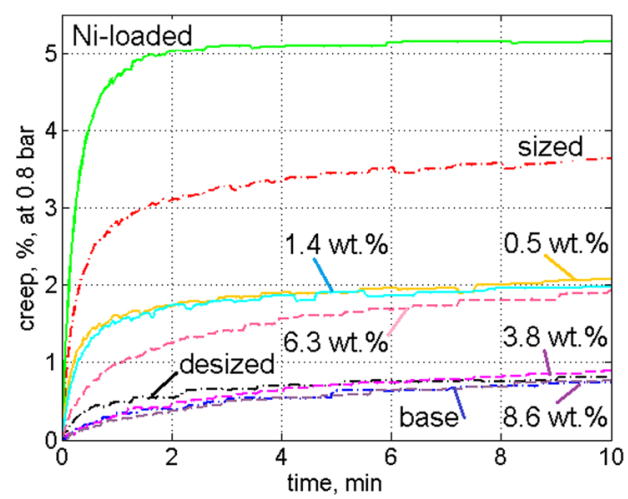

\section{Bias extension}

The shearing behavior of fabrics is a crucial property in their 3D formability. To check this for the present materials, bias extension tests are performed using rectangular $\mathrm{W}=60 \mathrm{~mm}$ wide and $\mathrm{H}=210 \mathrm{~mm}$ long (between the clamps) specimens, Fig. 14a. A $6 \mathrm{~mm} / \mathrm{min}$ displacement rate is applied during $10 \mathrm{~min}$; then the cross-head is stopped, and the load relaxation curve is sampled during the next 10-20 min. The exception is the $42 \mathrm{wt}$ \% specimens which are extended during 5 min only, due to 1) high resistance of this material that could overload the test rig and 2) deviating deformed shape of these specimens appearing already at relatively small displacements, Fig. 14d. Other specimens are also not geometrically perfect at high displacements, due to local out-of-plane bending of extended fabric, Fig. 14c; however, in this case the imperfections are confined within local areas relatively far from the central part of the specimens.

Three dry specimens are tested for each configuration (base, "desized", three different CNFs wt.\%). Figure 15a shows typical original load vs. time curves, while Fig. $15 \mathrm{~b}$ shows the same normalized to the maximal load. As seen, there is significant stress relaxation after the extension is stopped (and this cannot be attributed to slip in the clamps not detected here). This effect decreases with increase of the amount of grafting; this is in agreement with the influence of CNFs on the creep behavior, Figs. 10a and 13a.

In Fig. 15b, the normalized curves have similar shapes, except for the $42 \mathrm{wt} . \%$ specimen. However, if other curves are normalized not to the maximal force but to their level achieved after $5 \mathrm{~min}$ (that is the max. test time for the 42 wt. $\%$ specimens), all the curves approach the common almost linear - trend, although the $42 \mathrm{wt} . \%$ case still shows a stiffer response (this way of data reduction is not shown in the figures).

The shear angle, $\gamma$, is calculated by two ways. The first one relies on a purely geometrical approach proposed in e.g. [1, $17,18]$,

$\gamma=\pi / 2-2 \theta=\pi / 2-2 \arccos ((H-W+d) / \sqrt{2}(H-W))$,

where $d$ is the load head displacement, $\mathrm{H}$ and $\mathrm{W}$ as defined in Fig. 14. This formula gives about $7 \mathrm{deg} . / \mathrm{min}$ deformation rate.

The second approach uses the actual position of the yarns. For this purpose, a white square is painted in the central part of the specimen, Fig. 14. This area (which deforms into a rhomb) is monitored by a camera equipped with a low-distortion lens, taking a picture every $10 \mathrm{~s}$. These pictures are analyzed in Matlab, to calculate the actual shear angle.
Fig. 11 Ratio of Vf for wet and dry fabrics, vs. pressure, between 1 and 2-3 cycles of compression
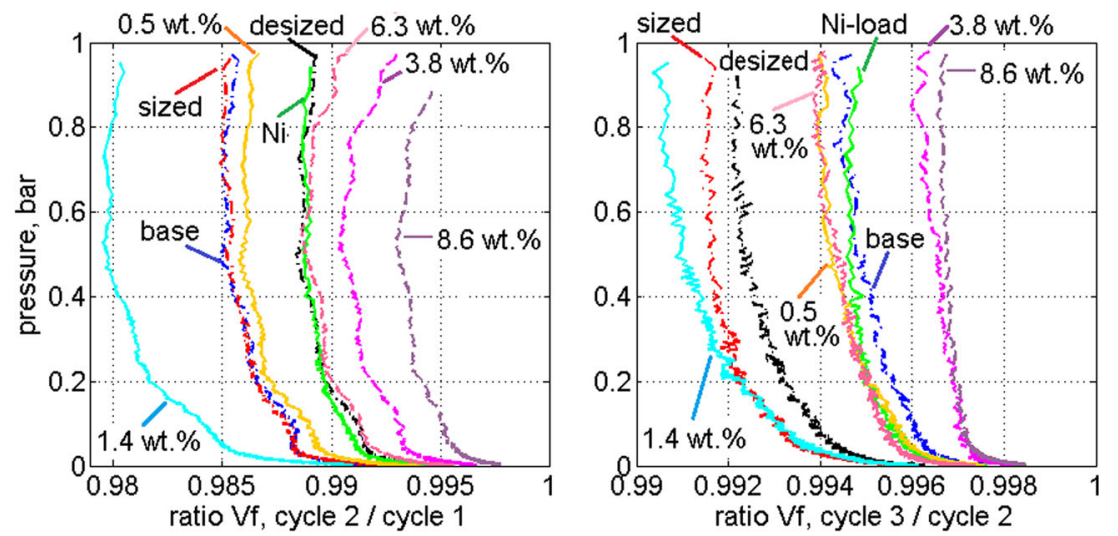


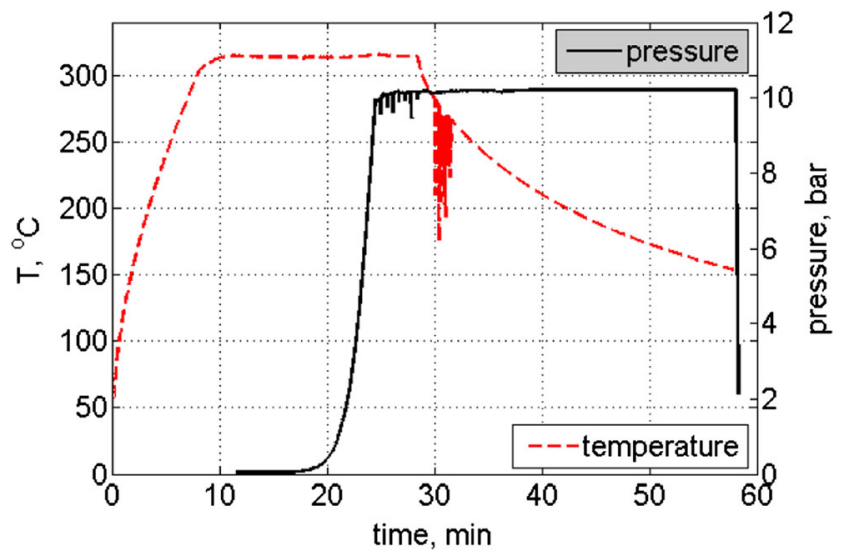

Fig. 12 Temperature-pressure regime for plate \#4, see also Table 2

Table 2 Production parameters for hot-pressed plates

\begin{tabular}{lllcc}
\hline Specimen & $\# 1$ & $\# 2$ & $\# 3$ & $\# 4$ \\
\hline CNF wt.\% & 0.0 (base) & 0.0 (base) & 0.9 & 3.8 \\
Max. Pressure, bar & 10 & 5 & 10 & 10 \\
Time at max. Pressure, min & 5 & 5 & 5 & 5 \\
Average thickness, mm & 3.08 & 3.36 & 3.13 & 3.43 \\
Average Vf, \% & 62.4 & 57.2 & 61.3 & 56.6 \\
\hline
\end{tabular}

The normalized shear force, $F_{n}$, is calculated according to $[1,17,18]$ as

$F_{n}(\gamma)=\left(k_{1} F(\cos (\gamma / 2)-\sin (\gamma / 2))-W F_{n}(\gamma / 2) \cos (\gamma / 2)\right) / k_{2} \cos (\gamma)$,

where $F$ is the load cell data, $\mathrm{k}_{1}=\mathrm{H} / \mathrm{W}-1, \mathrm{k}_{2}=2 \mathrm{H}-3 \mathrm{~W}$.

To use this implicit formula, the first two elements of vector $\mathrm{F}_{\mathrm{n}}(\gamma / 2)$ are approximated by

$F_{n 2}^{i=1}=k_{1} F_{1}(\cos (\gamma / 4)-\sin (\gamma / 4)) / k_{2} \cos (\gamma / 2)$, $F_{n 2}^{i=2}=\left(k_{1} F_{2}(\cos (\gamma / 4)-\sin (\gamma / 4))-W F_{n 2}^{i=1}(\cos (\gamma / 4)) / k_{2} \cos (\gamma / 2)\right.$.
For larger $i, \mathrm{~F}_{\mathrm{n}}(\gamma / 2)$ is simply constructed from the previous elements of the vector.

Typical results are shown in Fig. 16a (real force values) and $16 \mathrm{~b}$ (normalized shear force) vs. the shear angle. It is seen again that even a relatively small grafting (3.8. wt.\%) results in a significant increase of the in-plane shear stiffness, as well as in a prominent decrease of the "shear locking angle", Table 3. This "locking angle" is defined as the angle where the load curve slope increases, Fig. 16a.

The same figure and table reveal that, while the shear angle is calculated with a significant error (if compare with the measured value) at large strains (roughly - after reaching the "locking angle"), effect of this discrepancy on the normalized shear force is much milder.

As also shown in Table 3, the discrepancy between the calculated and measured shear angles vary little with the CNF wt.\% (0.86-1.05 after 5 min of loading, $0.65-0.71$ after other $5 \mathrm{~min}$ ). This means that this discrepancy is more a result of geometrical nonlinearity than the result of material specifics.

\section{Friction}

Friction plays an important role in forming processes. Therefore, a simple slope test is performed to measure the dry friction coefficient between the fabric-aluminium and fabric-fabric pairs, where either the base or grafted (6.5 wt.\%) fabric is used. A flat plate is covered with the fabric; "bare" or "covered" aluminium block is placed on it (thus mimicking the tool-ply or ply-ply friction), and then the slope of the plate is changed gradually. The onset of slippage is detected visually, and the corresponding slope, $\alpha$, is measured and recalculated into the friction coefficient $\operatorname{as} \tan (\alpha)$. The test setup is illustrated in Fig. 17.

Since the interest is to estimate the effect of grafting and not to measure "exact" properties, no special care is given for the block surface pre-treatment. It is just a thick piece of aluminium (of unknown type) having a flat, smooth, and degreased surface without scratches (but the surface roughness is not measured). The same side of the block is always used to contact the fabric, and orientation of the block is always the same.
Fig. 13 Results from the hotpressing with PPS foils. a Vf vs. pressure ("base dry" specimen was tested up to 20 bar; dots show Vf-pressure data from Table 2). b Creep behavior (inflection of curves at about $10 \mathrm{~min}$ corresponds to the onset of cooling) (a)

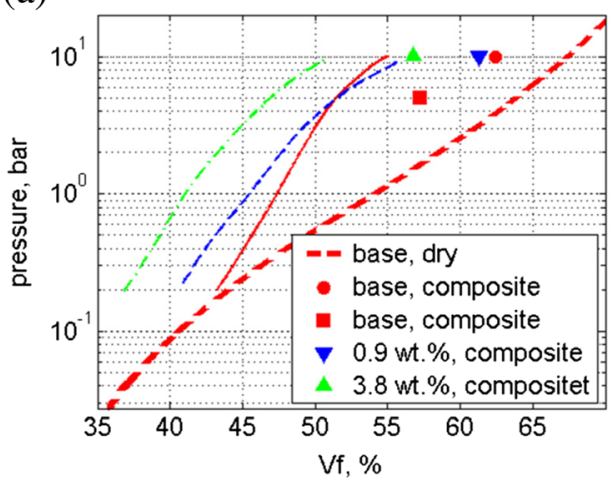

(b)

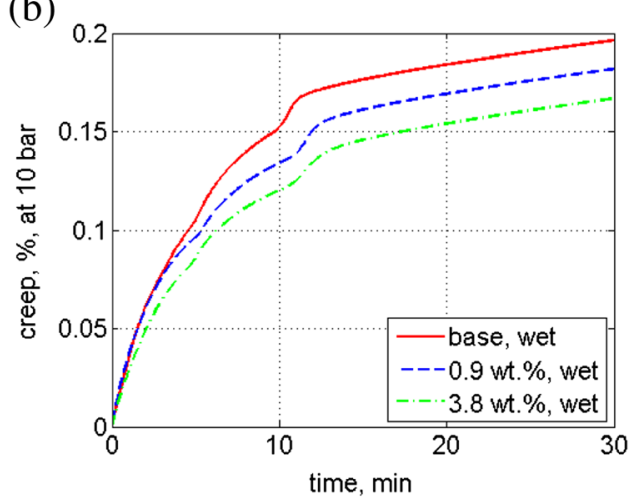


(a)

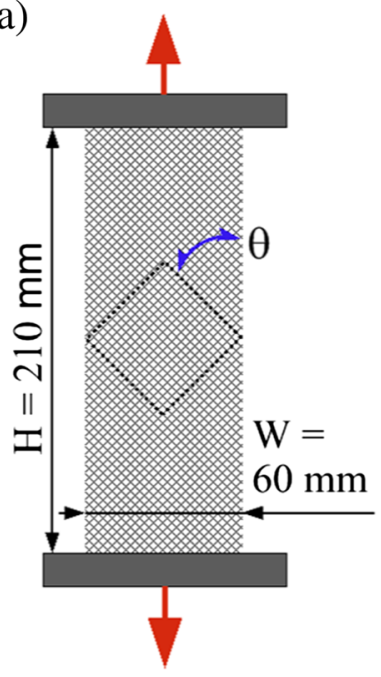

(b)

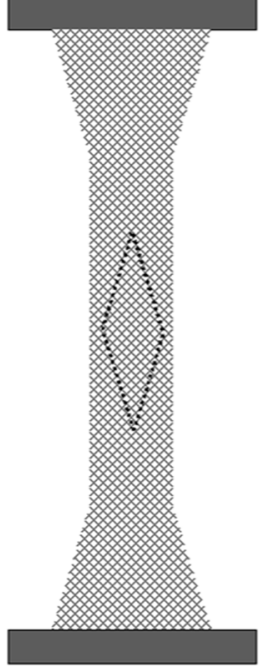

(c)

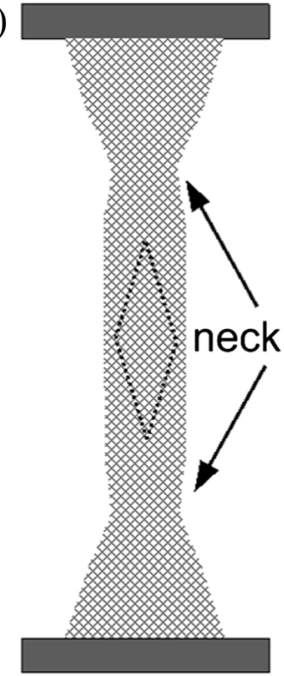

(d)

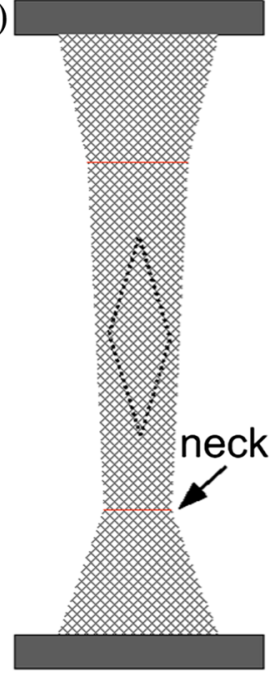

Fig. 14 Schematic of the specimens: (a) initial shape, (b) ideal deformed shape, (c) real shape with double "neck" (observed for a soft fabric), (d) real shape with single "neck" (observed for a rigid fabric). Dashed rhombs schematically illustrate the shear angle observed in the central region

Fig. 15 Typical load vs. time curves: original (a) or normalized (b) (a)

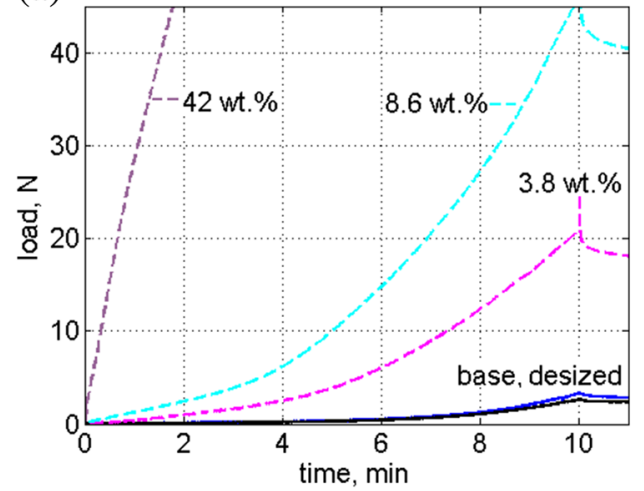

(b)

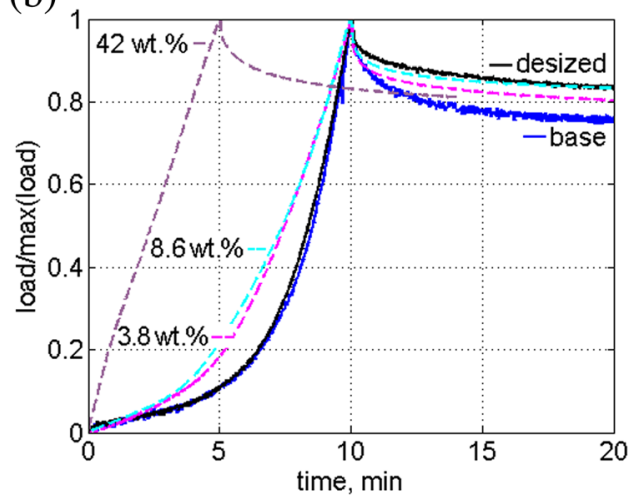

To measure the effect of contact pressure, additional mass is placed on the top of the block.

Different fabric orientations and their combinations are studied as shown symbolically in Fig. 18. Six measurements are performed and averaged for every case; the standard deviation is small (typically $0.01-0.02$ ), in spite of a nonautomated way of testing.
The left figure presents the simplest set of the orientations, when they depend on the single fabric lay-up only; as mentioned above, the aluminium block orientation is kept the same. The smaller friction occurs when the warp yarns (and, therefore, the most of longitudinal fibres at the fabric surface) are positioned along the weight movement. The largest friction happens when the fabric is rotated by $90^{\circ}$, and the
Fig. 16 Typical load vs. shear angle curves: original (a) or normalized (b, angles calculated with Eqs. (1) and (2) are shown with lines, measured angles are shown with dots) (a)

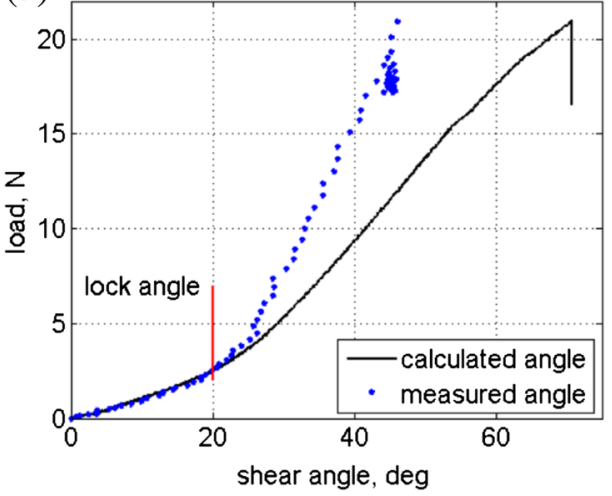

(b)

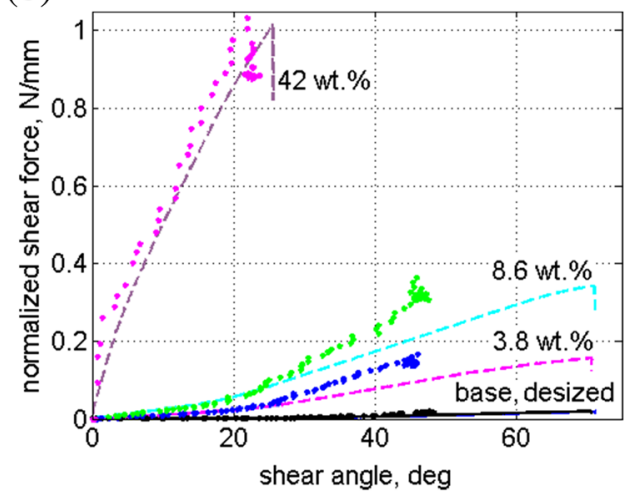


Table 3 Shear stiffness test results

\begin{tabular}{lccccc}
\hline Fabric: & Desized & Base & 3.8 wt.\% & 8.6 wt.\% & 42 wt.\% \\
\hline Lock angle,deg & 26 & 26 & 20 & 17 & $\mathrm{n} / \mathrm{a}$ \\
Ratio of $\gamma$ at $5 \mathrm{~min}$ & 1.05 & 0.96 & 0.89 & 0.93 & 0.86 \\
Ratio of $\gamma$ at $10 \mathrm{~min}$ & 0.71 & 0.67 & 0.65 & 0.65 & - \\
Ratio of $\mathrm{F}_{\mathrm{n}}$ at $5 \mathrm{~min}$ & 0.84 & 0.93 & 0.95 & 0.93 & 1.01 \\
Ratio of $\mathrm{F}_{\mathrm{n}}$ at $10 \mathrm{~min}$ & 1.03 & 1.04 & 1.06 & 1.06 & - \\
\hline
\end{tabular}

The ratios of $\gamma$ mean the measured angles divided by the calculated angles, Eq. (1), after 5 or 10 min of loading. The ratios of $F_{n}$ denote ratios of normalized shear forces, Eq. (2), calculated for the aforementioned shear angles

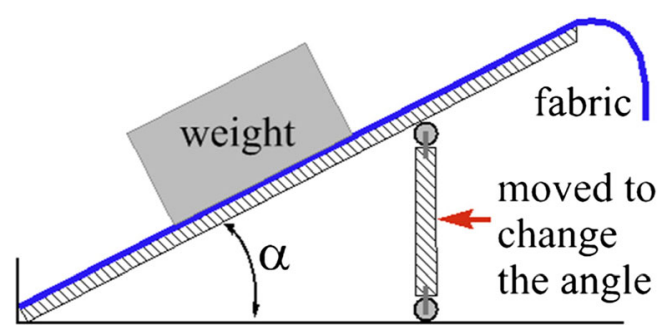

Fig. 17 Schematic of "tool-ply" friction test. In the "ply-ply" case, the weight is also dressed with the fabric

transverse fibres predominate at the interface. The $45^{\circ}$ case should be in between the two first cases but the present tests show it to give either the smallest or the largest friction.

The right figure reveals results for the ply-ply friction, and the number of possible orientational combinations is larger. The first expectation is to see the lowest friction in the case when the warp yarns are parallel in both fabrics, while in reality this case results in an intermediate values, probably due to closer contact of parallel fibres.
The results show that the $6.5 \mathrm{wt} . \%$ grafting increases the friction coefficient in most of cases but not always, Fig. 18 (right). Reasons of this discrepancy are not clear to the authors (the observed macroscopic friction coefficient originates from a rather complex interactions of nesting, interlocking and adhesion at the meso- and microlevels).

The measurements were performed for two contact pressures (assuming homogeneity of the pressure profile), to reveal a possible effect of nesting. The first assumption is that the nesting (if any) should increase the contact surface and, therefore, should increase the friction coefficient too. However, Fig. 18 shows a small but statistically constant decrease of this parameter along with increased contact pressure. This is because the contact surface increases less than the normal load. Therefore, since the friction force can be considered proportional to the contact surface, the friction coefficient decreases with the normal load [19].

\section{Discussion}

Results of this study can be outlined as the following:

- The deformability of the tested carbon woven reinforcement is seriously affected after growing the CNFs. The effect can be either positive (easier deformability) or negative (stiffer fabric), depending on the amount (wt.\%) of grown CNFs;

- A relatively high CNF wt.\% (say, more than 2\%) makes the fabric stiffer and this can be a disadvantage in practical applications. For example, a poorer compressibility can lower the achievable $\mathrm{Vf}$ for vacuum infusion or increase the pressure requirements in the hot pressing or autoclave. Another example is a stiffer response to shear deformations that can cause earlier wrinkles in $3 \mathrm{D}$ parts;
Fig. 18 Coulomb friction coefficient (mean values) for fabric-aluminium (a) and fabricfabric (b) pairs. Red symbols base fabric, blue symbols 6.5 wt. $\%$ grafted fabric. The fabric (fibre) orientation is denoted as " " for $0^{\circ}=$ warp (longitudinal fibres are dominant at the interface), "-" for $90^{\circ}=$ weft (transverse fibres are dominant), " " for $+45^{\circ}$, and " " for $-45^{\circ}$
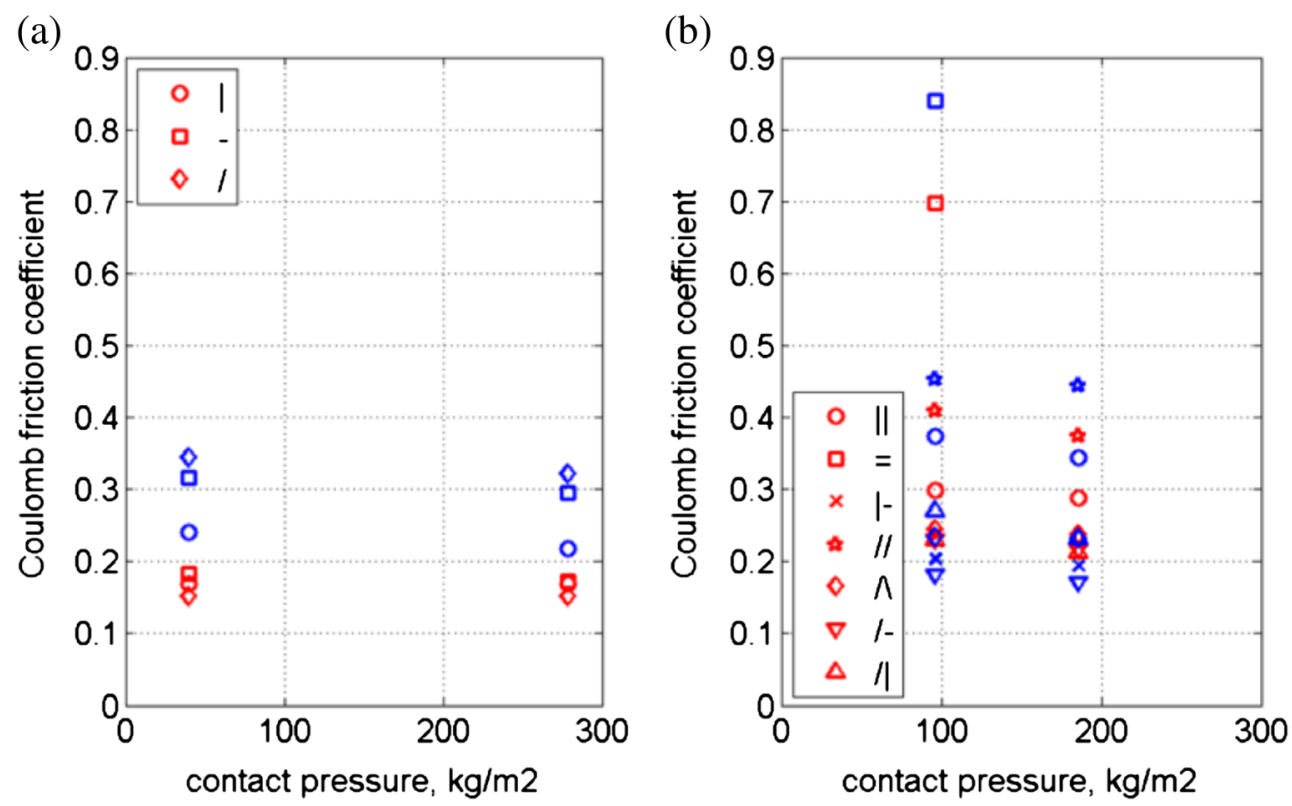
- However for a low (say, less than 1.5 wt.\%) amount of grown CNFs, the deformability can be even better than for the original fabric. This is due to removing (by a chemical treatment and high temperature) of the original sizing from the fibres. In the same time such a small amount of CNFs is not yet enough to compensate the fabric softening. This effect (in combination with the fibre surface modification by CNFs) can be an advantage from the production point of view as well as for the resulting mechanical properties;

- Creep can play a prominent role in approaching the target fibre volume fraction, especially in combination with high-viscosity resins, although the grown CNFs seem to limit this effect;

- The fabric-aluminum and fabric-fabric friction coefficient is affected by the grafting only slightly, being increased in the most of the cases. Lower friction is detected for several fabric-fabric orientations.

\section{Conclusions}

The deformability of a carbon textile (with in-situ grown carbon nano-fibres, or with a thermal treatment) was studied using bending, compression, in-plane shear, and friction tests. The main conclusion is that the grown CNFs can either degrade the deformability or improve it, with about a $1 \%$ mass fraction of grown CNFs as the threshold. Therefore a particular production case requires adjustment of the optimal mass fraction, to compromise decreased deformability of the preform and increased strength of a final composite part.

Acknowledgements The work was performed within Transforce ('Transverse Reinforcement of Carbon Fibre Composites with Carbon NanoFibres') project primary funded by STW, The Netherlands. The used fabric was kindly provided by TenCate Advanced Composites B.V.

Vitaly Koissin is very thankful to Mr. Bo Cornelissen (University of Twente) and to Prof. Stepan Lomov (K.U.Leuven) for valuable discussions. Mr. Gert Jan Nevenzeland Mr. Ruben Lubkemann (University of Twente) are gratefully acknowledged for their help in specimen preparation and testing. Mr. Mark Smithers and Mrs. Laura Vargas (ibid) are gratefully acknowledged for their help with SEM images.

\section{Compliance with ethical standards}

Conflict of interest The authors declare that they have no conflict of interest.

Open Access This article is distributed under the terms of the Creative Commons Attribution 4.0 International License (http:// creativecommons.org/licenses/by/4.0/), which permits unrestricted use, distribution, and reproduction in any medium, provided you give appropriate credit to the original author(s) and the source, provide a link to the Creative Commons license, and indicate if changes were made.

\section{References}

1. Cao J et al (2008) Characterization of mechanical behavior of woven fabrics: experimental methods and benchmark results. Compos Part A 39:1037-1053

2. Downs WB, Baker RTK (1995) Modification of the surface properties of carbon fibers via the catalytic growth of carbon nanofibers. J Mater Res 10:625-633

3. Qian $\mathrm{H}$ et al (2010) Carbon nanotube-based hierarchical composites: a review. J Mater Chem 20:4751-4762

4. Shafi Ullah K, Jang-Kyo K (2011) Impact and delamination failure of multiscale carbon nanotube-fiber reinforced polymer composites: a review. Int J Aeronaut Space Sci 12:115-133

5. Al-Saleh MH, Sundararaj U (2011) Review of the mechanical properties of carbon nanofiber/polymer composites. Compos Part A 42: 2126-2142

6. Lomov SV et al (2010) Compressibility of CNT-grafted fibrous reinforcements: a theory. Int J Mater Form 3:627-630

7. Lomov SV, Gorbatikh L, Verpoest I (2011) A model for the compression of a random assembly of carbon nanotubes. Carbon 49: 2079-2091

8. Lomov SV et al (2011) Compressibility of carbon woven fabrics with carbon nanotubes/nanofibres grown on the fibres. Compos Sci Technol 71:315-325

9. Aravand MA et al (2016) Effect of "fuzzy" fiber morphology on the internal geometry of textile composites characterized by microcomputed tomography. Compos Part A 88:295-304

10. Lomov SV, Molnár K (2016) Compressibility of carbon fabrics with needleless electrospun PAN nanofibrous interleaves. Express Polym Lett 10:25-35

11. Kotanjac $\check{Z}$ et al (2015) Synthesis of carbon nanofibers on large woven cloth. C-J Carbon Res 1:2-15

12. Torayca ${ }^{\circledR}$ T300 Technical Data Sheet No. CFA-002. Available online: http://www.toraycfa.com/pdfs/T300JDataSheet.pdf (Accessed on 8 Apr 2016)

13. TenCate patent US $7,252,726 \mathrm{~B} 2$

14. Koissin V et al (2016) Carbon nanofibers grown on large woven cloths: morphology and properties of growth. C-J Carbon Res 2: 19-34

15. Koissin V, New evaluation method for pierce cantilever test results. In: proc. of TexComp-11 conference, 16-20 September 2013, Leuven, Belgium

16. Koissin V, Warnet LL, Akkerman R (2013) Delamination in CFRP composites improved with in-situ grown nanofibres. Eng Fract Mech 101:140-148

17. Lee $\mathrm{W}$ et al (2008) Bias-extension of woven composite fabrics. Int $\mathbf{J}$ Mater Form Suppl 1:895-898

18. Launay $\mathrm{J}$ et al (2008) Experimental analysis of the influence of tensions on in plane shear behaviour of woven composite reinforcements. Compos Sci Technol 68:506-515

19. Cornelissen B et al (2013) Frictional behaviour of high performance fibrous tows: a contact mechanics model of tow-metal friction. Wear 305:78-88 